\title{
Hypoalbuminemia after pancreaticoduodenectomy does not predict or affect short-term postoperative prognosis
}

Wei Xut ${ }^{\dagger}$ Xiangqun Peng ${ }^{\dagger}$ and Bo Jiang ${ }^{*}$

\begin{abstract}
Background: Hypoalbuminemia (HA) is a risk factor for the complications following pancreaticoduodenectomy (PD). This study aimed to explore the factors that affect HA following PD and evaluate the influence of HA on the short-term postoperative prognosis.

Methods: Total 163 patients who underwent PD and met inclusion criteria were subdivided into two groups according to the status of HA. The relationships of postoperative albumin (ALB) level and exogenous ALB infusion with postoperative responses and complications were assessed by correlation analysis.

Results: Preoperative ALB $\geq 35.0 \mathrm{~g} / \mathrm{L}$ and postoperative complication grade were factors influencing HA after PD. Correlation analysis demonstrated significant negative correlation of postoperative ALB level with white blood cell (WBC) count and neutrophil count. Postoperative exogenous ALB infusion positively correlated with blood urea nitrogen, creatinine, complication grade, postoperative intraperitoneal hemorrhage and pancreatic fistula. No significant differences were observed between the complications and30-day mortality rates with and without postoperative HA.

Conclusions: HA after PD should not be considered as an indicator but rather a result of poor prognosis. WBCs, especially neutrophils, are involved in reducing postoperative ALB level. Infusion of exogenous ALB to maintain ALB $>30 \mathrm{~g} / \mathrm{L}$ could not improve clinical outcomes.
\end{abstract}

Keywords: Abdominal surgery, Outcomes, Complication, Pancreaticoduodenectomy, Hypoalbuminemia, Albumin

\section{Background}

Pancreaticoduodenectomy (PD) is a complicated abdominal surgical procedure and postoperative mortality for PD has decreased to $3-5 \%$ with improvements in the surgical technique and perioperative management [1-3]. However, the complication rate following PD is still as high as $30-60 \%[4,5]$. Hypoalbuminemia (HA) is a risk

\footnotetext{
* Correspondence: jiangbohnsrmyy01@163.com

'Wei Xu, Xiangqun Peng and Bo Jiang contributed equally to this work. Department of Hepatobiliary Surgery, Hunan Provincial People's Hospital, The First Hospital Affiliated with Hunan Normal University, No. 61 West Jiefang Road, Changsha 410005, China
}

factor for pancreatic fistula and other complications such as bleeding, intra-abdominal abscess formation, and multiple organ failure, thereby increasing postoperative mortality rate $[6,7]$. Multiple studies have reported that HA may increase the incidence of complications and mortality, prolong ICU admission and hospital stay, and increase the cost of medical resources $[8,9]$. Infusion of exogenous albumin (ALB) was reported to improve clinical outcomes in several studies, although other studies reached the opposite conclusion [10-14]. Despite inconsistent conclusions, clinicians often aim to

(c) The Author(s). 2020 Open Access This article is licensed under a Creative Commons Attribution 4.0 International License, which permits use, sharing, adaptation, distribution and reproduction in any medium or format, as long as you give appropriate credit to the original author(s) and the source, provide a link to the Creative Commons licence, and indicate if changes were made. The images or other third party material in this article are included in the article's Creative Commons licence, unless indicated otherwise in a credit line to the material. If material is not included in the article's Creative Commons licence and your intended use is not permitted by statutory regulation or exceeds the permitted use, you will need to obtain permission directly from the copyright holder. To view a copy of this licence, visit http://creativecommons.org/licenses/by/4.0/. The Creative Commons Public Domain Dedication waiver (http://creativecommons.org/publicdomain/zero/1.0/) applies to the data made available in this article, unless otherwise stated in a credit line to the data. 
increase postoperative ALB level to a so-called "safe" range to improve patient condition. However, the cause and effect relationship between HA and postoperative prognosis is still widely disputed. As a challenging abdominal surgical procedure, PD comprises various types of abdominal surgeries including organ resection and digestive tract reconstruction. It is of great clinical significance to investigate the factors that influence postoperative HA and related complications in PD compared with other abdominal operations.

The postoperative ALB level is affected by the recovery process, complications, and daily fluid therapy management strategy, such as the ratio of crystalloid/colloidal fluid. Therefore, this study aimed to explore the factors influencing postoperative $\mathrm{HA}$ and assess the relationship between ALB changes and complications after PD. In addition, we aimed to investigate the role of exogenous albumin in the prognosis of patients after PD.

\section{Methods \\ Patients}

All subjects have given written informed consent and the study protocol was approved by Ethics Committee of Hunan Provincial People's Hospital, The First Hospital Affiliated with Hunan Normal University (Approval No. 2702, Date 2015-12-04). In this prospective study, 238 consecutive patients who had undergone PD during two-year period between January 2016 and January 2018 in our hospital were initially enrolled. Patients were excluded if they underwent: (1) pancreatogastrostomy reconstruction; (2) Braun anastomosis in digestive tract reconstruction; and (3) total laparoscopic PD and conversion to open procedure, to avoid the interference of additional procedures on patient prognosis. All patients were subdivided into two groups according to the postoperative status of HA: HA group and non-HA group. HA was defined by serum ALB level $\leq 30 \mathrm{~g} / \mathrm{L}$ until the 14th day after operation independent of the supplementation with exogenous ALB.

\section{Preoperative examination and radiographic assessment}

Routine blood tests, biochemical examination, measurement of carbohydrate antigen 19-9 (CA19-9), abdominal ultrasound, computed tomography $(\mathrm{CT})$, magnetic resonance imaging (MRI)/magnetic resonance cholangiopancreatography (MRCP), and endoscopic ultrasound (EUS) were performed before operation. Only preoperatively latest examination results were adopted in this study. Routine blood tests, liver and kidney function tests, electrolyte levels, coagulation, and C-reactive protein (CRP) level, amylase levels in blood and drainage, and lipase detection were examined on the $1,3,5,7,10$ and 14days after operation. Postoperative abdominal ultrasound and/or CT were applied to evaluate ascites routinely.

\section{Operative procedure}

Classical PD was performed for all patients. Briefly, after kocherization of the pancreatic head and duodenum, the pancreas was divided anteriorly and to the left of the superior mesenteric vein and portal vein. En bloc removal of each specimen involved distal stomach resection at $10-40 \%$ and removal of all of the duodenum, gallbladder, and common bile duct (transected at the cystic duct confluence level) as well as approximately $10-20 \mathrm{~cm}$ of the proximal jejunum distal to the ligament of Treitz, with resection of the head, neck, and uncinate process of the pancreas. The scope of lymphadenectomy was dependent on the nature of the lesions and observations on operative exploration. Types of pancreaticojejunostomy reconstruction comprised of duct-to-mucosa endto-side pancreaticojejunostomy with internal or external stent, invagination pancreaticojejunostomy with internal or external stent, or pancreaticojejunostomy without stent application. The stenting silicone tube could be guided externally through the jejunal loop (external stent) or cut short and left in the jejunal loop (internal stent).

\section{Definition and classification of complications}

Postoperative complications were graded according to the Clavien-Dindo criteria [15]. Postoperative pancreatic fistula (POPF) was defined by an amylase concentration in the drainage fluid exceeding three times the upper limit of normal value or pancreatic intestinal anastomotic rupture on imaging [16]. Delayed gastric emptying (DGE) was defined by: 1) nasogastric tube decompression $\geq 3$ days and meeting one of the following standards: a) vomiting after nasogastric tube removal, b) medication needed to improve gastrointestinal motility at 10 days postoperatively, c) replacement of the gastric tube, and d) inability to tolerate a solid diet by 7 days after operation; or 2) nasogastric tube decompression $\geq 10$ days and meeting two of the explained standards [17]. Postpancreatectomy hemorrhage (PPH) cases were divided into early stage hemorrhage within $24 \mathrm{~h}$ after operation and late stage hemorrhage occurring more than $24 \mathrm{~h}$ after operation [18]. Ascites was detected by postoperative abdominal ultrasound or CT after exclusion of pancreatic fistula, biliary fistula, anastomotic fistula, or chylous ascites, and the ascites severity was further graded as mild when the depth of effusion was $<3 \mathrm{~cm}$ or the daily drainage was $<50 \mathrm{ml}$, as middle when the depth of effusion was $3-5 \mathrm{~cm}$ or the daily drainage was between 50 and $500 \mathrm{ml}$, and large when the depth of effusion was $\geq 5 \mathrm{~cm}$ or the daily drainage exceeded $500 \mathrm{ml}$. Hyperbilirubinemia was 
defined by blood TBIL $\geq 171.0 \mu \mathrm{mol} / \mathrm{L}$, postoperative TBIL elevation to $\geq 171.0 \mu \mathrm{mol} / \mathrm{L}$ in patients with a preoperative TBIL $<171.0 \mu \mathrm{mol} / \mathrm{L}$, or postoperative elevation to $\geq 171.0 \mu \mathrm{mol} / \mathrm{L}$ after transient decline. Infectious complications included incision infection, intra-abdominal abscess, anastomotic fistula, pneumonia, and septicemia [19].

\section{Postoperative management}

All patients received standard care for postoperative management. Somatostatin ( $3 \mathrm{mg} / \mathrm{Q} 12 \mathrm{~h})$ was administered to all patients by transfusion with a micro-infusion pump through postoperative day 7 or extended according to postoperative complications. A gastric tube was routinely placed preoperatively and removed after the recovery of gastrointestinal function. The drainage tube was removed if there was no evidence of biliary fistula or pancreatic fistula. Total parenteral nutrition was used for patients with an inadequate diet and stopped when the patients could tolerate at least half of their oral diet. An exogenous ALB preparation (Baxter Healthcare, Deerfield, USA) was adopted to maintain ALB level $>30$ $\mathrm{g} / \mathrm{L}$. The endpoint was short-term analysis of early postoperative results.

\section{Statistical analysis}

Measurement data are depicted as median (minimum maximum). Rates were compared by Pearson $x^{2}$ test, Fisher's test, or Wilcoxon rank sum test. The medians were compared by the Mann-Whitney $U$ test. Binary logistic regression was used for postoperative univariate and multivariate analyses. Canonical correlation analysis was used to evaluate the relationship of postoperative ALB level and infused amount with indicators and complications. A standardized canonical coefficient (SCC) > 0.5 was considered relevant. All statistical analysis was performed using PASW Statistics software 18.0 (SPSS Inc., Chicago, USA). $P<0.05$ was considered significant.

\section{Results}

\section{Patient characteristics}

A total of 238 patients underwent PD between January 2016 and January 2018 in our hospital. Of these, 13 cases with pancreatogastrostomy reconstruction, 15 cases with Braun anastomosis in the digestive tract reconstruction, 43 cases with total laparoscopic PD, and 4 cases that required conversion to laparotomy were excluded. The remaining 163 patients were enrolled in the present study, including 35 cases of lower common bile duct cancer, 49 cases of duodenal papilla carcinoma, 49 cases of pancreatic head carcinoma, 17 cases of periampullary carcinoma, and 13 cases with an inflammatory mass at the head of the pancreas. On the postoperative pathology, 140 cases were confirmed to be adenocarcinoma, 2 cases were adenosquamous carcinoma, 5 cases were mucinous adenocarcinoma, 12 cases involved an inflammatory mass, 2 cases were cystadenocarcinoma, 1 case was sarcoma, and 1 case involved a retention cyst. The ratio of men to women was 1.3:1, and the average patient age was 57.8 years (range, $36-79$ years; Table 1 ).

\section{Postoperative complications}

A total of $89(54.6 \%)$ cases had no complications. According to classification based on the Clavien- Dindo criteria, the percentages of all patients who experienced grade I to grade $\mathrm{V}$ complications were grade I $22.1 \%$ (36/163), grade II $11.0 \%$ (18/163), grade IIIa $4.3 \%$ (7/ $163)$, grade IIIb $3.7 \%(6 / 163)$, grade IVa $1.2 \%(2 / 163)$, grade IVb $0 \%(0 / 163)$, and grade $\mathrm{V} 3.1 \%(5 / 163)$, respectively. The 30-day in-hospital mortality was two cases in HA group and one case in non-HA group, and 60-day in-hospital mortality was three cases in HA group and two cases in non-HA group, showing no significant difference.

The most common complication was ascites (37.4\%), followed by infection (30.1\%), pancreatic fistula (18.4\%), and postoperative upper gastrointestinal tract hemorrhage (6.1\%). Five patients presented with early intraperitoneal hemorrhage, of which two cases underwent reoperation and three received conservative treatment, two cases were cured after conservative treatment, and one died due to liver and kidney dysfunction. Nine cases experienced late intraperitoneal hemorrhage, of which three cases received laparotomy after hemostatic failure, two cases underwent interventional embolization treatment, and four cases were treated with double-catheter irrigation and local application of hemostatic drugs.

The incidence of upper gastrointestinal hemorrhage was $6.1 \%(10 / 163)$, including one case that experienced early upper gastrointestinal bleeding within $12 \mathrm{~h}$ and was treated with hemostatic drugs and nine cases with late bleeding within $3 \sim 23$ days postoperatively that resolved after conservative treatment. Five patients presented with biliary fistula (3.1\%), including one case of both pancreatic fistula and late intraperitoneal hemorrhage that was treated by reoperation. The percentages of patients who experienced DGE of grades $\mathrm{B}$ and $\mathrm{C}$ were $1.8 \%(3 / 163)$ and $1.2 \%(2 / 163)$, respectively, and hyperbilirubinemia affected $6.13 \%(10 / 163)$ of the patients.

Most complications were evaluated as grade I, including incision site infection and urinary tract infection. Intra-abdominal abscess formation (1.8\%) was the most common type of infection among cases with grade II complications.

\section{Factors influencing HA after PD}

Univariate analysis showed that the following five factors were associated with postoperative HA: preoperative 
Table 1 Analysis of potential factors as predictors of postoperative HA

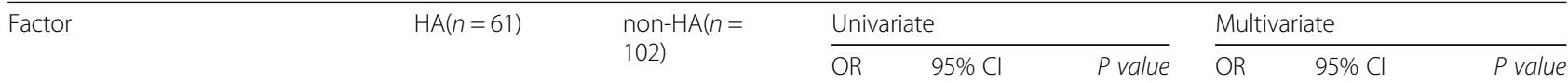

\section{Patient characteristics}

Gender

Male
Female
Age (years)
Weight loss $>5 \mathrm{~kg}$
Yes
No
BMI(kg/m² $)^{\text {a }}$
Diabetes mellitus

No

Yes

Acute pancreatitis

$\begin{array}{ll}\text { No } & 59 \\ \text { Yes } & 2\end{array}$

Chronic pancreatitis

$$
\text { No }
$$$$
\text { Yes }
$$

19

Preoperative biliary drainage

$$
\text { No }
$$$$
\text { Yes }
$$

58

3

Preoperative ALB usage

No
Yes

\section{Preoperative ALB usage ${ }^{a}(g)$}

$\operatorname{WBC}\left(\times 10^{9} /\right)^{a}$

$\operatorname{NEUT}\left(\times 10^{9} / \mathrm{L}\right)^{\mathrm{a}}$

$\operatorname{LYMP}\left(\times 10^{9} / \mathrm{L}\right)^{\mathrm{a}}$

$\operatorname{CRP}(\mathrm{mg} / \mathrm{L})^{\mathrm{a}}$

$\mathrm{RBC}\left(\times 10^{12} / \mathrm{L}\right)^{\mathrm{a}}$

$\mathrm{Hb}(\mathrm{g} / \mathrm{L})^{\mathrm{a}}$

$\mathrm{HCT}(\%)^{a}$

$\operatorname{PLT}\left(\times 10^{9} / \mathrm{L}\right)^{\mathrm{a}}$

$\mathrm{PT}(\mathrm{s})^{\mathrm{a}}$

$\operatorname{APTT}(s)^{a}$

$\Pi(s)^{a}$

INR $^{\mathrm{a}}$

$\operatorname{ALT}(\mathrm{U} / \mathrm{L})^{\mathrm{a}}$

AST $(U / L)^{a}$

$\operatorname{ALP}(\mathrm{U} / \mathrm{L})^{\mathrm{a}}$

58

3

$14.7 \pm 4.2$

$6.6 \pm 2.3$

$4.6 \pm 2.0$

$1.2 \pm 0.5$

$16.1 \pm 5.4$

$3.8 \pm 0.5$

$116.3 \pm 17.6$

$33.6 \pm 4.9$

$206.7 \pm 90.4$

$11.5 \pm 1.7$

$28.2 \pm 4.7$

$19.8 \pm 2.9$

$0.9 \pm 0.1$

$112.3 \pm 95.2$

$96.0 \pm 65.1$

$509.3 \pm 390.1$

TBIL $\geq 171.1(\mu \mathrm{mol} / \mathrm{L})$

$$
\text { No }
$$

Yes
34

27
62

40

$56.6 \pm 9.7$

31

71

$21.6 \pm 3.3$

97

5

98

4

57

45

94

8

97

5

$34.5 \pm 9.4$

$6.3 \pm 2.1$

$4.3 \pm 1.9$

$1.4 \pm 0.6$

$19.7 \pm 7.6$

$3.9 \pm 0.6$

$119.8 \pm 19.4$

$36.7 \pm 4.6$

$224.6 \pm 94.6$

$11.3 \pm 1.6$

$27.4 \pm 4.9$

$19.4 \pm 2.5$

$0.9 \pm 0.2$

$162.3 \pm 78.8$

$115.6 \pm 94.7$

$498.4 \pm 374.0$

76

26
1

0.604

0.317-1.149

0.124

1.036

$1.000-1.074 \quad 0.051$

1

$0.621 \quad 0.284-1.357 \quad 0.232$

1.000

$0.884-1.131 \quad 0.996$

1

$1.021 \quad 0.235-4.433 \quad 0.978$

1

$0.845 \quad 0.150-4.757 \quad 0.848$

1

$1.021 \quad 0.235-4.433 \quad 0.978$

1

$0.618 \quad 0.158-2.427 \quad 0.491$

1

$0.276 \quad 0.046-1.638 \quad 0.157$

$0.999 \quad 0.975-1.023 \quad 0.910$

$1.074 \quad 0.926-1.247 \quad 0.346$

$\begin{array}{lll}1.087 & 0.923-1.280 & 0.319\end{array}$

$0.631 \quad 0.337-1.182 \quad 0.151$

$\begin{array}{lll}1.011 & 0.968-1.056 & 0.617\end{array}$

$0.720 \quad 0.402-1.290 \quad 0.270$

$0.990 \quad 0.973-1.007 \quad 0.263$

$\begin{array}{lll}0.863 & 0.719-1.035 & 0.112\end{array}$

$\begin{array}{lll}0.998 & 0.994-1.002 & 0.247\end{array}$

$1.089 \quad 0.891-1.330 \quad 0.406$

$1.032 \quad 0.964-1.104 \quad 0.371$

$1.062 \quad 0.937-1.203 \quad 0.346$

$\begin{array}{lll}1.763 & 0.180-7.228 & 0.626\end{array}$

$0.997 \quad 0.995-1.000 \quad 0.057$

$0.997 \quad 0.993-1.001 \quad 0.165$

$\begin{array}{lll}1.000 & 0.999-1.001 & 0.861\end{array}$
$2.214 \quad 1.117-4.390 \quad 0.023$

$0.997 \quad 0.267-3.720$

0.997 
Table 1 Analysis of potential factors as predictors of postoperative HA (Continued)

\begin{tabular}{|c|c|c|c|c|c|c|c|c|}
\hline \multirow[t]{2}{*}{ Factor } & \multirow[t]{2}{*}{$\mathrm{HA}(n=61)$} & \multirow{2}{*}{$\begin{array}{l}\text { non- } \mathrm{HA}(n= \\
102)\end{array}$} & \multicolumn{3}{|c|}{ Univariate } & \multicolumn{3}{|c|}{ Multivariate } \\
\hline & & & $\mathrm{OR}$ & $95 \% \mathrm{Cl}$ & $P$ value & $\mathrm{OR}$ & $95 \% \mathrm{Cl}$ & $P$ value \\
\hline $\mathrm{TP}(\mathrm{g} / \mathrm{L})^{a}$ & $59.4 \pm 2.8$ & $61.7 \pm 6.0$ & 0.905 & $0.755-1.084$ & 0.279 & & & \\
\hline \multicolumn{9}{|l|}{ Preoperative ALB $\geq 35.0$ (g/L) } \\
\hline No & 25 & 26 & 1 & & & & & \\
\hline Yes & 36 & 76 & 0.480 & $0.241-0.955$ & 0.037 & 0.447 & $0.210-0.952$ & 0.037 \\
\hline $\mathrm{GLB}(\mathrm{g} / \mathrm{L})^{\mathrm{a}}$ & $25.7 \pm 3.5$ & $24.9 \pm 3.7$ & 1.062 & $0.853-1.323$ & 0.588 & & & \\
\hline $\mathrm{PA}(\mathrm{mg} / \mathrm{L})^{\mathrm{a}}$ & $201.8 \pm 95.9$ & $187.2 \pm 71.7$ & 1.002 & $0.997-1.007$ & 0.366 & & & \\
\hline $\operatorname{AMY}(U / L)^{a}$ & $85.0 \pm 59.2$ & $145.6 \pm 106.3$ & 0.996 & $0.988-1.004$ & 0.347 & & & \\
\hline CA19-9 $(\mathrm{U} / \mathrm{mL})^{\mathrm{a}}$ & $648.5 \pm 133.7$ & $622.8 \pm 147.2$ & 1.000 & $1.000-1.000$ & 0.941 & & & \\
\hline \multicolumn{9}{|l|}{ Site of Lesion } \\
\hline Distal common bile duct & 18 & $17(16.7)$ & 1 & & 0.262 & & & \\
\hline Duodenal papilla & $17(27.9)$ & $32(31.4)$ & 0.472 & $0.193-1.155$ & 0.100 & & & \\
\hline Pancreatic head & $21(34.4)$ & $41(40.2)$ & 0.561 & $0.234-1.342$ & 0.194 & & & \\
\hline Ampullary & $5(8.2)$ & 12(11.8) & 0.747 & $0.557-1.003$ & 0.052 & & & \\
\hline \multicolumn{9}{|l|}{ Intra-Operative factors } \\
\hline Operation time $(\mathrm{h})^{\mathrm{a}}$ & $7.6 \pm 1.5$ & $7.8 \pm 1.7$ & 0.923 & $0.754-1.129$ & 0.435 & & & \\
\hline Estimated Blood loss $(\mathrm{ml})^{a}$ & $591.5 \pm 451.2$ & $497.4 \pm 311.1$ & 1.001 & $1.000-1.002$ & 0.128 & & & \\
\hline pRBC transfusion $(U)^{a}$ & $1.7 \pm 0.6$ & $1.5 \pm 0.5$ & 1.081 & $0.881-1.325$ & 0.455 & & & \\
\hline Plasma transfusion(ml) & $237.5 \pm 80.3$ & $132.8 \pm 50.9$ & 1.002 & $1.000-1.004$ & 0.009 & 1.002 & $1.000-1.003$ & 0.057 \\
\hline Total liquid volume(ml) & $5829.2 \pm 1431.7$ & $6171.3 \pm 1544.2$ & 1.000 & $1.000-1.000$ & 0.275 & & & \\
\hline Ratio of crystal fluid/colloid fluid & $1.8 \pm 0.5$ & $2.3 \pm 0.6$ & 0.219 & $0.046-1.040$ & 0.056 & & & \\
\hline \multicolumn{9}{|l|}{ Pancreatic gland texture } \\
\hline Soft & 36 & 58 & 1 & & & & & \\
\hline Hard & 25 & 44 & 0.603 & $0.322-1.129$ & 0.114 & & & \\
\hline \multicolumn{9}{|l|}{ Pancreatic duct diameter $>0.3 \mathrm{~cm}$} \\
\hline No & 27 & 25 & 1 & & & 1 & & \\
\hline Yes & 34 & 77 & 0.425 & $0.215-0.839$ & 0.014 & 0.819 & $0.369-1.815$ & 0.622 \\
\hline \multicolumn{9}{|l|}{ PJ-R Type } \\
\hline IPJ (external stenting) & 49 & 81 & 1 & & 0.219 & & & \\
\hline DMPJ (external stenting) & 0 & 2 & - & - & - & & & \\
\hline DMPJ (internal stenting) & 4 & 14 & 1.189 & $0.449-3.152$ & 0.728 & & & \\
\hline IPJ (no stenting) & 5 & 4 & 5.833 & $0.953-7.717$ & 0.056 & & & \\
\hline IPJ (internal stenting) & 3 & 1 & 1.460 & $0.914-2.330$ & 0.113 & & & \\
\hline \multicolumn{9}{|l|}{ Combined portal vein resection } \\
\hline No & 60 & 100 & 1 & & & & & \\
\hline Yes & 1 & 2 & 1.180 & $0.105-3.296$ & 0.893 & & & \\
\hline \multicolumn{9}{|l|}{ Resectional margin status } \\
\hline $\mathrm{RO}^{\mathrm{b}}$ & 53 & 91 & 1 & & 0.697 & & & \\
\hline R1 & 5 & 5 & 1.692 & $0.468-6.124$ & 0.423 & & & \\
\hline R2 & 3 & 6 & 1.046 & $0.559-1.956$ & 0.889 & & & \\
\hline
\end{tabular}

\section{Pathological characteristics and postoperative complications}

Pathology

$\begin{array}{lllll}\text { Adenocarcinoma } & 59(96.7) & 85(83.3) & 1 & 0.949 \\ \text { Mucinous adenocarcinoma } & 0(0) & 5(4.9) & - & -\end{array}$


Table 1 Analysis of potential factors as predictors of postoperative HA (Continued)

\begin{tabular}{|c|c|c|c|c|c|c|c|c|}
\hline \multirow[t]{2}{*}{ Factor } & \multirow[t]{2}{*}{$\mathrm{HA}(n=61)$} & \multirow{2}{*}{$\begin{array}{l}\text { non-HA(n= } \\
102)\end{array}$} & \multicolumn{3}{|c|}{ Univariate } & \multicolumn{3}{|c|}{ Multivariate } \\
\hline & & & $\mathrm{OR}$ & $95 \% \mathrm{Cl}$ & $P$ value & $\mathrm{OR}$ & $95 \% \mathrm{Cl}$ & $P$ value \\
\hline Inflammation mass & 2 & 10 & 0.754 & $0.565-1.005$ & 0.054 & & & \\
\hline Sarcoma & 0 & 1 & - & - & - & & & \\
\hline Retention cyst & 0 & 1 & - & - & - & & & \\
\hline \multicolumn{9}{|l|}{ Tumor Differentiation } \\
\hline Inflammation & 2 & 11 & 1 & & 0.570 & & & \\
\hline High & 26 & 45 & 0.900 & $0.356-2.274$ & 0.824 & & & \\
\hline Moderate & 23 & 27 & 1.033 & $0.592-3.974$ & 0.379 & & & \\
\hline Poor & 10 & 19 & 1.167 & $0.758-1.797$ & 0.484 & & & \\
\hline \multicolumn{9}{|l|}{ Clavien-Dindo grade } \\
\hline 0 & 28 & 61 & 1 & & 0.262 & 1 & & 0.173 \\
\hline । & 11 & 25 & 0.472 & $0.193-1.155$ & 0.100 & 0.620 & $0.105-3.656$ & 0.597 \\
\hline$\|$ & 12 & 6 & 0.561 & $0.234-1.342$ & 0.194 & 1.127 & $0.179-7.085$ & 0.899 \\
\hline IIla & 2 & 5 & 3.765 & $0.897-9.296$ & 0.067 & 1.582 & $0.316-7.927$ & 0.577 \\
\hline$\| l l b$ & 2 & 4 & 3.351 & $1.641-6.845$ & 0.001 & 4.317 & $1.479-12.602$ & 0.007 \\
\hline IVa & 2 & 0 & - & - & - & - & - & - \\
\hline $\mathrm{IVb}$ & 0 & 0 & - & - & - & - & - & - \\
\hline V & 4 & 1 & 1.654 & $1.186-2.306$ & 0.003 & 1.605 & $1.097-2.349$ & 0.015 \\
\hline
\end{tabular}

Abbreviations: $O R$ odds ratio, $B M I$ body mass index, $T B I L$ total bilirubin, $A L T$ alanine aminotransferase, $A S T$ aspartate aminotransferase, $A L P$ alkaline phosphatase, $T P$ total protein, GLB globulin, $A L B$ albumin, $P A$ prealbumin, $A M Y$ amylase, CRP C-reactive protein, $R B C$ red blood cell count, WBC white blood cell count, NEUT neutrophil count, $L Y M P$ lymphocyte count, $H b$ hemoglobin, $P L T$ platelet count, $H C T$ hematocrit, $P T$ prothrombin time, $A P T T$ activated partial thromboplastin time, $\Pi$ thrombin time, INR international normalized ratio, CA19-9 carbohydrate antigen $19-9, p R B C$ packed red blood cell, $P J-R$ pancreaticojejunostomy reconstruction, DMPJ duct-to-mucosa end-to-side pancreaticojejunostomy, IPJ invagination pancreaticojejunostomy

a expressed as Mean $\pm \mathrm{SD}$;

${ }^{\mathrm{b}}$ The resectional margin status in non-malignant disease was classified and analyzed as R0

TBIL $\geq 171.1 \mu \mathrm{mol} / \mathrm{L}$, preoperative ALB $\geq 35.0 \mathrm{~g} / \mathrm{L}$, classification of complications, main pancreatic duct inner diameter $>0.3 \mathrm{~cm}$, and intraoperative plasma transfusion (Table 1). Multivariate analysis showed that preoperative ALB $\geq 35.0 \mathrm{~g} / \mathrm{L}$ and the complication grade were the factors significantly influencing HA after PD (Table 1).

\section{Correlations of preoperative ALB level and ALB infusion with various indicators}

As shown in Table 2, liver function, inflammatory reaction index, coagulation, renal function and electrolyte index, postoperative complications, digestive tract anastomotic leakage, postoperative RBC and plasma transfusion, ascites, and diarrhea and somatostatin application exhibited correlation with ALB level and ALB infusion amount. No coagulation index was relevant with ALB level and ALB infusion amount. Surgical treatment of complication, early and late postoperative intraperitoneal hemorrhage were significantly correlated with HA. RBC and plasma transfusion may not affect serum ALB level. High request of RBC transfusion, such as severe complication, may generate the clinical circumstance of ALB infusion to correct HA. ALB level showed no correlation with ascites formation and volume. ALB infusion amount exhibited weak positive correlation with ascites, and weak negative correlation with positive germiculture in abdominal drainage. Somatostatin application and diarrhea after operation showed no correlation.

\section{Comparison of postoperative parameters between HA and non-HA groups}

No significant difference was observed in the rates of complications between the HA and non-HA groups (Table 3). Moreover, no differences were observed in the total amount of ALB infused $(P=0.186)$, ALB infusion frequency $(P=0.365)$, or postoperative 30,60 -day fatality rate $(P=0.557,0.066$, respectively) between the two groups. The classes of complications showed significant difference between the two groups $(P=0.020)$.

\section{Discussion}

In this study, we analyzed the factors influencing HA after PD and the effects of HA on short-term prognosis. Multivariate analysis showed that preoperative ALB $\geq 35.0 \mathrm{~g} / \mathrm{L}$ and the classification of postoperative complications significantly affected the occurrence of HA after $\mathrm{PD}$, but the underlying mechanism is still elusive. We postulate that ALB level may indicate inflammation status in the body which may affect postoperative HA, but further studies are needed to confirm it. 


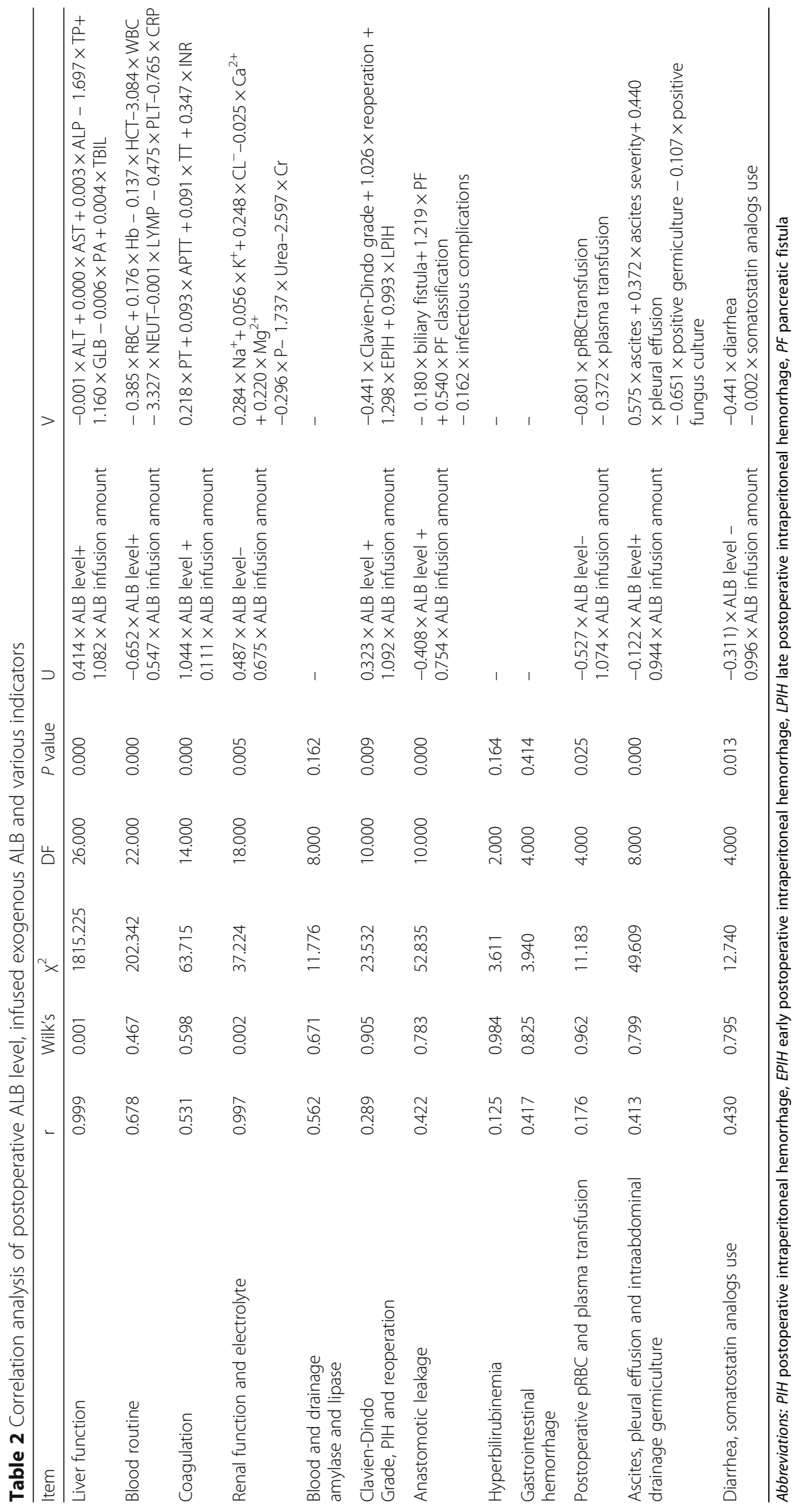


Table 3 Comparison of postoperative parameters between HA and non-HA groups

\begin{tabular}{|c|c|c|c|}
\hline Parameters & $\mathrm{HA}(n=61)$ & non-HA(n=102) & $P$ value $^{a}$ \\
\hline EPPH & 3 & 2 & 0.360 \\
\hline EPPH treatment & & & 1.000 \\
\hline Non-surgical therapy & 2 & 1 & \\
\hline Reoperation & 1 & 1 & \\
\hline $\mathrm{LPPH}$ & 4 & 5 & 0.729 \\
\hline \multicolumn{4}{|l|}{ LPPH treatment } \\
\hline Conservation & 1 & 3 & 0.539 \\
\hline Intervention & 1 & 1 & \\
\hline Surgery & 2 & 1 & \\
\hline POPF & 12 & 18 & 0.747 \\
\hline POPF grade & & & $0.076^{b}$ \\
\hline A & 6 & 14 & \\
\hline B & 2 & 3 & \\
\hline C & 4 & 1 & \\
\hline Biliary fistula & 1 & 4 & 0.651 \\
\hline DGE Grade C & 2 & 3 & 1.000 \\
\hline Hyperbilirubinemia & 4 & 6 & 1.000 \\
\hline Infection complications & 20 & 29 & $0.557^{c}$ \\
\hline \multicolumn{4}{|l|}{ Infection site } \\
\hline Incision & 15 & 15 & $0.079^{c}$ \\
\hline Lung & 1 & 0 & \\
\hline Intra-abdominal & 4 & 14 & \\
\hline Ascites & 26 & 35 & $0.289^{c}$ \\
\hline \multicolumn{4}{|l|}{ Ascites severity } \\
\hline Mild & 14 & 25 & $0.157^{c}$ \\
\hline Middle & 12 & 10 & \\
\hline Postoperative delirium & 3 & 1 & 0.148 \\
\hline Cardiovascular events & 1 & 0 & 0.374 \\
\hline Liver dysfunction & 2 & 0 & 0.139 \\
\hline Upper gastrointestinal hemorrhage & 5 & 5 & 0.503 \\
\hline Lower gastrointestinal hemorrhage & 1 & 0 & 0.374 \\
\hline Multiorgan dysfunction & 2 & 1 & 0.557 \\
\hline Clavien-Dindo classification & & & $0.020^{b}$ \\
\hline 0 & 28 & 61 & \\
\hline I & 11 & 25 & \\
\hline$\|$ & 12 & 6 & \\
\hline IIla & 2 & 5 & \\
\hline Illb & 2 & 4 & \\
\hline IVa & 2 & 0 & \\
\hline $\mathrm{IVb}$ & 0 & 0 & \\
\hline V & 4 & 1 & \\
\hline Reoperation & 2 & 4 & 1.000 \\
\hline Postoperative ALB infusion dosage $(\mathrm{g})$ * & $169.0 \pm 126.1$ & $115.6 \pm 87.9$ & $0.186^{d}$ \\
\hline Postoperative ALB infusion frequency* & $10.7 \pm 6.9$ & $5.81 \pm 4.0$ & $0.365^{d}$ \\
\hline
\end{tabular}


Table 3 Comparison of postoperative parameters between HA and non-HA groups (Continued)

\begin{tabular}{|c|c|c|c|}
\hline Parameters & $\mathrm{HA}(n=61)$ & non- $\mathrm{HA}(n=102)$ & $P$ value $^{a}$ \\
\hline Somatostatin application (day) ${ }^{*}$ & $9.3 \pm 4.2$ & $10.1 \pm 3.8$ & $0.215^{d}$ \\
\hline Postoperative pRBC transfusion $(\mathrm{U}) *$ & $4.3 \pm 3.1$ & $4.0 \pm 1.9$ & $0.003^{d}$ \\
\hline Postoperative plasma transfusion $(\mathrm{ml}) *$ & $869.0 \pm 633.8$ & $545.9 \pm 373.1$ & $0.086^{d}$ \\
\hline Hospital stay (days) ${ }^{*}$ & $30.0 \pm 13.6$ & $29.2 \pm 13.4$ & $0.658^{d}$ \\
\hline 30-day Mortality & 2 & 1 & 0.557 \\
\hline 60-day Mortality & 4 & 1 & 0.066 \\
\hline
\end{tabular}

$a$ : Fisher's exact test; $b$ : Wilcoxon rank sum test; $c$ : Pearson $x^{2}$ test; $d$ : Mann-Whitney $U$ test

Abbreviations: EPPH early postpancreatectomy hemorrhage, LPPH Late postpancreatectomy hemorrhage

* expressed as Mean \pm SD

Correlation analysis showed that postoperative ALB level was only negatively correlated with WBC and neutrophil counts. The amount of exogenous ALB infused postoperatively was negatively correlated with TP but positively correlated with BUN, Cr, complications requiring surgical intervention, early intraperitoneal hemorrhage, late intraperitoneal hemorrhage and pancreatic fistula. Importantly, the incidences of complications and the 30-day mortality rates did not differ significantly between $\mathrm{HA}$ group and non-HA group.

About $20-40 \%$ of patients present with HA after selected operations [20]. Exogenous ALB infusion may correct postoperative $\mathrm{HA}$ and maintain intravascular colloid osmotic pressure. However, the endothelial glycocalyx (EG) layer plays a role in intra- and extracellular liquid exchange, reabsorption of interstitial fluid may not occur even if exogenous ALB is infused to improve plasma colloid osmotic pressure [21]. In addition, although ALB is a key component of the EG layer, only $25 \%$ of physiological concentration of ALB can maintain the complete EG layer, and EG layer remains functional even with an ALB level of $10 \mathrm{~g} / \mathrm{L}$ [22]. Thus, exogenous ALB infusion is not recommended to improve and maintain the function of the EG layer.

ALB level after PD and infusion of exogenous ALB exhibited no relationship with HCT in this study, indicating that hemodilution was not the main reason for HA, consistent with previous report [20]. The integrity and function of the EG layer suffer damage during inflammatory response [23]. Excessive inflammation leads to the release of many inflammatory mediators and increased number of blood leukocytes, especially neutrophils, contributing to EG layer barrier dysfunction, leukocyte adhesion to the vascular wall and increased vascular permeability [24].

This study revealed that postoperative ALB level negatively correlated with postoperative inflammation indexes, such as WBC count, neutrophil absolute value, and CRP level. The correlation of CRP with postoperative ALB level was lower than that of the WBC and neutrophil counts, suggesting that WBC and neutrophils, especially neutrophils, have specific roles in the ALB decline after PD, probably via damage to the EG layer. We speculated that the surgery itself, as the initial stimulus of inflammation, led to an increase in the WBC count, especially in neutrophils. The release, activation and dynamic changes of different subgroups of neutrophils contributed to the damage and subsequent dysfunction of the EG layer. This may explain why infusion of exogenous ALB cannot reliably improve HA in some patients and even correcting ALB level $>30 \mathrm{~g} / \mathrm{L}$ does not effectively prevent serious complications. Furthermore, such damage and functional loss of the EG layer may not be resolved while inflammation subsides during recovery, as reflected by a decrease in the neutrophil count, because serum ALB level slowly returns to the normal range over a relatively long time after the operation.

Anastomotic fistula after PD, including bile leakage, pancreatic leakage, and intra-abdominal abscess formation, is the most serious complication that significantly increases postoperative mortality, and may become aggravated as late as the 8-12th day [25]. This study demonstrated that a reduction in postoperative ALB may not affect the occurrence of pancreatic fistula. Moreover, infusion of exogenous ALB could not prevent the occurrence of pancreatic fistula. However, exogenous ALB infusion was positively correlated with surgical treatment of postoperative complications and postoperative intraperitoneal hemorrhage, especially the early postoperative intraperitoneal hemorrhage. Early hemorrhage after PD can be caused by the operative technique or coagulation dysfunction, which usually requires abdominal laparotomy to stop the bleeding [1]. For the patients who underwent reoperation, two operations in a short period induced excessive inflammation, leading to ALB decrease. In this case, the clinician may use exogenous ALB to correct HA. However, late postoperative bleeding usually occurs in 1-3 weeks after surgery. The cause of this complication is complex and may be related to peripancreatic blood vessel corrosion secondary to pancreatic leakage, aneurysm formation, or ulceration of 
anastomotic site [26]. Generally, postoperative bleeding can be stopped upon interventional therapy or endoscopic treatment under local anesthesia. Our results suggest that postoperative $\mathrm{RBC}$ or plasma transfusion did not improve ALB level.

Based on our results, the strategy for correcting HA after PD is to block or rapidly end the acute phase of the inflammatory response and protect or restore the EG layer, although the clinical benefit of this approach is questionable. In addition, careful attention to perioperative nutritional support can help patients pass through the acute stress reaction period, even though parenteral nutrition has been shown to seldom correct ALB concentration to normal level [27].

There are several limitations in this study. First, PD procedures were performed by different physicians, and thus the amounts of fluid infused daily and the ratio of crystal and colloid varied. The impact of such differences on the occurrence of postoperative complications is difficult to evaluate. Second, exogenous ALB preparation used in this study was $20 \%$ concentration, and the impact of other concentrations of ALB on HA after PD remains unclear. Third, the variety of diagnoses may contribute to possible bias in our conclusion. Fourth, we only evaluated short-term outcomes of patients after PD and exogenous ALB infusion. Fifth, the sample size is not big enough. Further multi-center randomized prospective studies with long-term follow-up are needed.

\section{Conclusions}

Our study provides further evidence that HA should not be considered as an indicator but rather a result of poor prognosis. WBC, especially neutrophils, exhibit unique role in reducing postoperative ALB level. Infusion of exogenous ALB to maintain ALB $>30 \mathrm{~g} / \mathrm{L}$ has no clinical benefit for patients after PD.

\section{Abbreviations}

ALB: Albumin; HA: Hypoalbuminemia; PD: Pancreaticoduodenectomy

\section{Acknowledgements}

We thank the patients for their participation in this study.

\section{Authors' contributions}

BJ conceived the study. WX and XP collected and analyzed the data. All authors read and approved the final manuscript.

\section{Funding}

This study was supported by Hunan Provincial Natural Science Foundation of China (Grant No. 2018JJ6016). The funding body has no role in the design of the study and collection, analysis, and interpretation of data and in writing the manuscript.

\section{Availability of data and materials}

All data used in the study are available from correspondence author Bo Jiang upon reasonable request.

\section{Ethics approval and consent to participate}

This study was approved by Ethics Committee of Hunan Provincial People's Hospital
(The First Hospital Affiliated with Hunan Normal University) and all patients provided written informed consent.

\section{Consent for publication}

Not applicable.

\section{Competing interests}

The authors declare that they have no competing interests.

Received: 20 October 2019 Accepted: 6 April 2020

Published online: 15 April 2020

\section{References}

1. Zovak M, Mužina Mišić D, Glavčić G. Pancreatic surgery: evolution and current tailored approach. Hepatobiliary Surg Nutr. 2014;3:247-58.

2. Büchler MW, Wagner M, Schmied BM, Uhl W, Friess H, Z'graggen $K$. Changes in morbidity after pancreatic resection: toward the end of completion pancreatectomy. Arch Surg. 2003;138:1310-4 discussion 1315.

3. McPhee JT, Hill JS, Whalen GF, Zayaruzny M, Litwin DE, Sullivan ME, Anderson FA, Tseng JF. Perioperative mortality for pancreatectomy: a national perspective. Ann Surg. 2007;246:246-53.

4. Topal B, Fieuws S, Aerts R, Weerts J, Feryn T, Roeyen G, Bertrand C, Hubert C, Janssens M, Closset J. Belgian Section of Hepatobiliary and Pancreatic Surgery, Pancreaticojejunostomy versus pancreaticogastrostomy reconstruction after pancreaticoduodenectomy for pancreatic or periampullary tumours: a multicentre randomised trial. Lancet Oncol. 2013; 14:655-62.

5. Heeger K, Fendrich V, Waldmann J, Langer P, Kanngießer V, Bartsch DK. Reduced complication rate after modified binding purse-string-mattress sutures pancreatogastrostomy versus duct-to-mucosa pancreaticojejunostomy. Surgeon. 2013;11:246-52.

6. Malleo G, Pulvirenti A, Marchegiani G, Butturini G, Salvia R, Bassi C. Diagnosis and management of postoperative pancreatic fistula. Langenbeck's Arch Surg. 2014;399:801-10.

7. Hsieh WC, Aboud A, Henry BM, Omara M, Lindner J, Pirk J. Serum albumin in patients undergoing transcatheter aortic valve replacement: a metaanalysis. Rev Cardiovasc Med. 2019;20:161-9.

8. Lee Jl, Kwon M, Roh JL, Choi JW, Choi SH, Nam SY, Kim SY. Postoperative hypoalbuminemia as a risk factor for surgical site infection after oral cancer surgery. Oral Dis. 2015;21:178-84.

9. Kantor O, Talamonti MS, Stocker SJ, Wang CH, Winchester DJ, Bentrem DJ, Prinz RA, Baker MS. A graded evaluation of outcomes following Pancreaticoduodenectomy with major vascular resection in pancreatic Cancer. J Gastrointest Surg. 2016;20:284-92.

10. Mendez CM, McClain CJ, Marsano LS. Albumin therapy in clinical practice. Nutr Clin Pract. 2005;20:314-20.

11. Finfer S, Myburgh J, Bellomo R. Albumin supplementation and organ function. Crit Care Med. 2007;35:987-8.

12. Ertmer C, Kampmeier TG, Volkert T, Wolters H, Rehberg S, Morelli A, Schmidt $H$, Lange M, Boschin M, Van Aken H, Hahnenkamp K. Impact of human albumin infusion on organ function in orthotopic liver transplantation--a retrospective matched-pair analysis. Clin Transpl. 2015;29:67-75.

13. Yuan XY, Zhang CH, He YL, Yuan YX, Cai SR, Luo NX, Zhan WH, Cui J. Is albumin administration beneficial in early stage of postoperative hypoalbuminemia following gastrointestinal surgery?: a prospective randomized controlled trial. Am J Surg. 2008;196:751-5.

14. Mahkovic-Hergouth $\mathrm{K}$, Kompan L. Is replacement of albumin in major abdominal surgery useful? J Clin Anesth. 2011;23:42-6.

15. DeOliveira ML, Winter JM, Schafer M, Cunningham SC, Cameron JL, Yeo CJ, Clavien PA. Assessment of complications after pancreatic surgery: a novel grading system applied to 634 patients undergoing pancreaticoduodenectomy. Ann Surg. 2006;244:931-7 discussion 937-939.

16. Bassi C, Dervenis C, Butturini G, Fingerhut A, Yeo C, Izbicki J, Neoptolemos J, Sarr M, Traverso W, Buchler M. International Study Group on Pancreatic Fistula Definition, Postoperative pancreatic fistula: an international study group (ISGPF) definition. Surgery. 2005;138:8-13.

17. Wente MN, Bassi C, Dervenis C, Fingerhut A, Gouma DJ, Izbicki JR, Neoptolemos JP, Padbury RT, Sarr MG, Traverso LW, Yeo CJ, Büchler MW. Delayed gastric emptying (DGE) after pancreatic surgery: a suggested definition by the international study Group of Pancreatic Surgery (ISGPS). Surgery. 2007;142:761-8. 
18. Wente MN, Veit JA, Bassi C, Dervenis C, Fingerhut A, Gouma DJ, Izbicki JR, Neoptolemos JP, Padbury RT, Sarr MG, Yeo CJ, Büchler MW.

Postpancreatectomy hemorrhage (PPH): an international study Group of Pancreatic Surgery (ISGPS) definition. Surgery. 2007;142:20-5.

19. Moyes LH, Leitch EF, McKee RF, Anderson JH, Horgan PG, McMillan DC. Preoperative systemic inflammation predicts postoperative infectious complications in patients undergoing curative resection for colorectal cancer. Br J Cancer. 2009;100:1236-9.

20. Ryan AM, Hearty A, Prichard RS, Cunningham A, Rowley SP, Reynolds JV. Association of hypoalbuminemia on the first postoperative day and complications following esophagectomy. J Gastrointest Surg. 2007;11:1355-60.

21. Woodcock TE, Woodcock TM. Revised Starling equation and the glycocalyx model of transvascular fluid exchange: an improved paradigm for prescribing intravenous fluid therapy. Br J Anaesth. 2012;108:384-94.

22. Zausig YA, Chappell D, Becker BF, Potschka D, Busse H, Nixdorf K, Bitzinger D, Jacob B, Jacob M. The impact of crystalloidal and colloidal infusion preparations on coronary vascular integrity, interstitial oedema and cardiac performance in isolated hearts. Crit Care. 2013;17:R203.

23. Chappell $D$, Westphal $M$, Jacob $M$. The impact of the glycocalyx on microcirculatory oxygen distribution in critical illness. Curr Opin Anaesthesiol. 2009;22:155-62.

24. Claesson-Welsh L. Vascular permeability--the essentials. Ups J Med Sci. 2015; 120:135-43.

25. Nelson A, Berkestedt I, Schmidtchen A, Ljunggren L, Bodelsson M. Increased levels of glycosaminoglycans during septic shock: relation to mortality and the antibacterial actions of plasma. Shock. 2008:30:623-7.

26. de Castro SM, Kuhlmann KF, Busch OR, van Delden OM, Laméris JS, van Gulik TM, Obertop H, Gouma DJ. Delayed massive hemorrhage after pancreatic and biliary surgery: embolfigtion or surgery? Ann Surg. 2005;241: $85-91$.

27. Hülshoff A, Schricker T, Elgendy H, Hatzakorzian R, Lattermann R. Albumin synthesis in surgical patients. Nutrition. 2013;29:703-7.

\section{Publisher's Note}

Springer Nature remains neutral with regard to jurisdictional claims in published maps and institutional affiliations.

Ready to submit your research? Choose BMC and benefit from:

- fast, convenient online submission

- thorough peer review by experienced researchers in your field

- rapid publication on acceptance

- support for research data, including large and complex data types

- gold Open Access which fosters wider collaboration and increased citations

- maximum visibility for your research: over $100 \mathrm{M}$ website views per year

At $\mathrm{BMC}$, research is always in progress.

Learn more biomedcentral.com/submissions 